\title{
White dwarf cooling sequences
}

\section{Luminosity functions}

\author{
P. G. Prada Moroni ${ }^{1,2}$ and O. Straniero ${ }^{3}$ \\ 1 Dipartimento di Fisica "E. Fermi", University of Pisa, largo B. Pontecorvo 3, 56127 Pisa, Italy \\ 2 INFN, largo B. Pontecorvo 3, 56127 Pisa, Italy \\ e-mail: prada@df.unipi.it \\ 3 INAF - Osservatorio Astronomico di Collurania, via Maggini, 64100 Teramo, Italy \\ e-mail: straniero@te.astro.it
}

Received 23 December 2006 / Accepted 29 January 2007

\section{ABSTRACT}

\begin{abstract}
Context. Given the importance of white dwarfs (WDs) in many fields of modern astrophysics, the precise knowledge of the actual degree of accuracy of the associated theoretical predictions is a primary task. In the first paper of a series dedicated to the modeling of WD structure and evolution we discussed the limits of the available theoretical studies of cooling sequences.

Aims. In the present work we extend this analysis to isochrones and luminosity functions of WDs belonging to old stellar systems, like globular or old disk clusters. The discussion is focused on the most common DA, those with a CO core and an H-rich envelope. Methods. We discuss, in particular, the variation of the age derived from the observed WD sequence caused by different assumptions about the conductive opacity as well as that induced by changing the carbon abundance in the core.

Results. The former causes a global uncertainty of the order of $10 \%$ and the latter of about $5 \%$. We discuss different choices of the initial-to-final mass relation, which induces an uncertainty of $8 \%$ on the GC age estimate.
\end{abstract}

Key words. stars: white dwarfs - stars: luminosity function, mass function - stars: evolution

\section{Introduction}

In the last decade, impressive improvement in observational techniques made available a significative sample of cold and faint white dwarfs (WDs) both in the field (Bergeron et al. 2001) and in stellar clusters (Paresce et al. 1995; Cool et al. 1996; Renzini et al. 1996; Richer et al. 1997; Von Hippel \& Gilmore 2000; Zoccali et al. 2001; Hansen et al. 2002; De Marchi et al. 2004; Monelli et al. 2005). The growing amount of data prompted a renewed interest in theoretical studies of WD evolution, particularly for the latest stages when the core crystallizes and the stars approaches the Debye regime (Benvenuto \& Althaus 1999; Hansen 1999; Chabrier et al. 2000; Salaris et al. 2000; Prada Moroni \& Straniero 2002, hereafter Paper I).

Concerning the core composition, the theory of stellar evolution predicts three kinds of WDs: helium, carbon/oxygen $(\mathrm{C}-\mathrm{O})$, oxygen/neon/magnesium. The most common are, by far, those with a $\mathrm{C}-\mathrm{O}$ core, which represent the final stage of the evolution of single (non-interacting) stars with mass lower than about $7 M_{\odot}\left(M_{\text {up }}\right)$ and a lifetime shorter than the age of the Galaxy. The envelope composition is also very important for the evaluation of the cooling timescale. Mass loss, particularly during the AGB and post-AGB, may substantially erode the envelope. Moreover, the H-rich envelope can be substantially reduced in the bornagain evolution scenario (Iben et al. 1983; Iben \& MacDonald 1995; Herwig 2005). Nevertheless, the large majority of the observed WDs show an hydrogen-rich atmosphere (DA WDs), while only $20 \%$ of them are hydrogen-deprived (DB WDs, see e.g. Koester \& Chanmugam 1990). The present study deals with the most common WDs, namely the DA type having a $\mathrm{C}-\mathrm{O}$ core.

Here we are interested in the use of WDs as cosmic chronometers (Schmidt 1959) and standard candles
(Fusi Pecci \& Renzini 1979). Due to the intrinsic faintness of WD sequences in stellar clusters, only very recently have these tools become useful. Their application to the nearest globular clusters (GCs) provides age and distance estimates that are independent of those obtained by means of the classical methods (those based on the main sequence and horizontal branch stars), thus allowing us to further constrain the lower limit of the Universe's age (Hansen et al. 2002; De Marchi et al. 2004). One should, however, check the reliability of the theoretical cooling time predictions before adopting WDs as cosmic clocks. Notwithstanding the significant advances in the computation of the WD structure and evolution, there is still a sizeable uncertainty in the prediction of the cooling times of the old WDs (Montgomery et al. 1999; Salaris et al. 2000; Fontaine et al. 2001; Paper I). At low luminosity $\left(\log L / L_{\odot} \sim-5.5\right)$ the differences among the most recent models available in the literature reach about 4 Gyr (see e.g. Fig. 1 in Paper I). In Paper I we analyzed some of the main sources of uncertainty in the evolution of a $0.6 M_{\odot} \mathrm{C}-\mathrm{O}$ WD. In particular, we discussed uncertainties due to both the WD progenitor history and the adopted input physics.

The evolution of a WD can be roughly described as a cooling process where the luminosity is supplied by the heat content of the internal matter; thermonuclear energy generation is negligible, except during the very early phase. Thus, the evolution results from the balance between the thermal energy stored in the $\mathrm{C}-\mathrm{O}$ ions constituting the core (about $98 \%$ of the WD mass) and the energy transport through the He-rich mantel and the $\mathrm{H}$-rich envelope, the most opaque region of the star.

In Paper I, we showed that the factors that most severely limit the accuracy of theoretical predictions are the assumed conductive opacity in the partially degenerate regime, a condition that is 

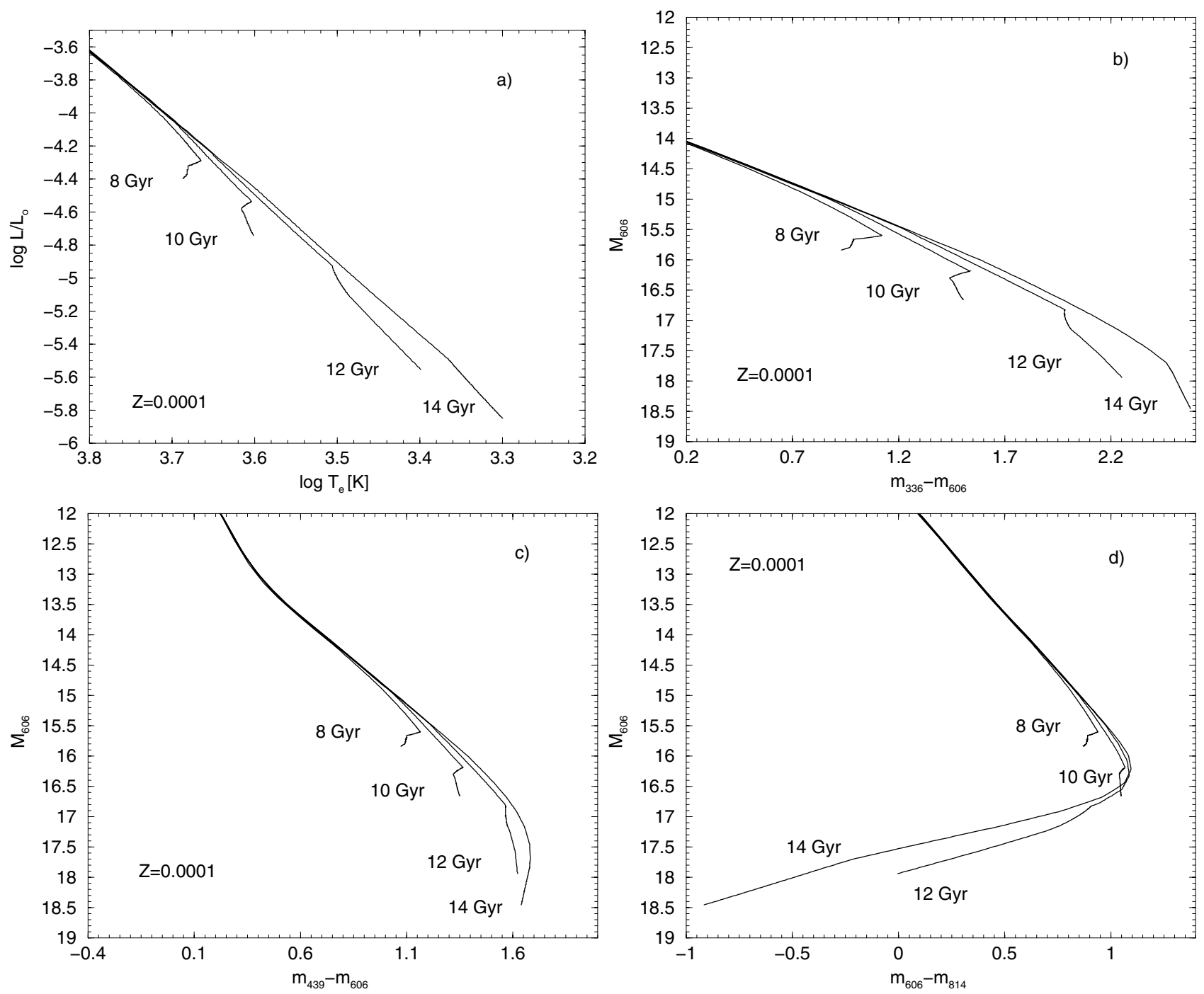

Fig. 1. WD isochrones for ages in the interval 8-14 Gyr in the theoretical plane, i.e. $\log L / L_{\odot}$ vs. $\log T_{\mathrm{e}}$ (panel a)); $V(606)$ vs. $U(336)-V(606)$ (panel b)); $V(606)$ vs. $B(439)-V(606)$ (panel c)); $V(606)$ vs. $V(606)-I(814)$ (panel d)). We adopted the HST/ACS transmission curves $F 336 W$ $($ broad $U), F 439 W($ broad $B), F 606 W(\operatorname{broad} V)$ and $F 814 W($ broad $I)$.

usually fulfilled in the outer layer of the core and in the He-rich mantel, and the amount of carbon and oxygen in the core. At $\log L / L_{\odot} \sim-5.5$, these two sources produce a total uncertainty on the predicted cooling age of about $27 \%$. However, the analysis reported in Paper I gives only a rough idea of the uncertainty in dating GCs with WDs, while a more precise estimate of this uncertainty should be based on WD luminosity functions instead of tracks of given mass, since this is the best WD chronometer. For this work we have calculated several grids of models of $\mathrm{C}-\mathrm{O}$ WDs with a hydrogen rich atmosphere, with mass in the range $0.5-0.9 M_{\odot}$, under different assumptions of the conductive opacity and the $\mathrm{C}-\mathrm{O}$ profile. The related isochrones and luminosity functions (LFs) are compared to reveal the main uncertainty factors.

\section{Dating WDs in old stellar clusters: the piling up in the luminosity function}

For old stellar systems, such as GCs, the best chronometer relying on WD evolution is their luminosity function instead of the related isochrones. Figure 1 shows WD isochrones for ages in the interval 8-14 Gyr in the theoretical plane $\left(\log L / L_{\odot}\right.$ vs. $\log T_{\mathrm{e}}$, panel a) and in three CM diagrams for different HST/ACS pass-bands, $F 336 W$ (broad $U$ ), $F 439 W($ broad $B$ ), $F 606 W($ broad $V)$ and $F 814 W($ broad $I)$. In the theoretical plane (panel a), there is a clear and unambiguous age dependent feature: the blue-turn of the WD isochrone, which plays the same role of clock pointer as the turn-off point in the main sequence phase. The luminosity of the blue-turn depends only on the evolutionary time scales, both of the WDs and of their progenitors. On the other hand, on the observational CM diagram, the situation is less clear, in fact the morphology of the old (>10 Gyr) WD isochrones significantly depends on the adopted pass-bands, particularly the luminosity of the blue-turn. In the $V(606)$ vs. $V(606)-I(814)$ diagram, commonly used in observations of cool WDs, the blue-turn is almost insensitive to the age for isochrones older than about 10 Gyr. This behavior is a consequence of the strong depletion of the IR flux in the emerging spectrum of cool $\left(T_{\mathrm{e}}<5000 \mathrm{~K}\right)$ DA WDs due to the collision induced absorption (CIA) of $\mathrm{H}_{2}-\mathrm{H}_{2}$ (see e.g. Bergeron et al. 1995). Since the latter blue shift is an atmospheric effect that reflects the departure of the emergent spectrum from the black body appearance, its extension depends sensitively on the wavelength of the transmission curves of the filter adopted in the observations. The blue 
shift will be much larger in the $V-I$ color than in the $B-V$, because the CIA is the main opacity source in the IR region of the spectrum. Such an occurrence explains why in the $V$ vs. $V-I$ plane the blue-hook luminosity of old WD isochrones is essentially insensitive to the age and the blue-tail of the 14 Gyr WD isochrone is brighter than the $12 \mathrm{Gyr}$ one. The degeneracy of the position of the blue-turn with age and the necessity to accurately model the CIA opacity sources in the WD model atmospheres severely reduce the effectiveness of the WD isochrones in dating old stellar systems such as galactic GCs. A much firmer estimate relies on star counts, i.e. luminosity functions, which directly reflect the evolutionary time scales.

Figure 2 shows the WD LFs for 12,13, 14 Gyr. These and the following WD LFs have been computed with Monte Carlo simulations that distribute 50000 WDs along the related isochrones. This figure clearly illustrates the potential of WD LFs in dating old stellar systems. Note that the peak of the luminosity function shifts by about 0.3 mag per Gyr in the $V$ band, while the main sequence turn-off luminosity, the clock classically used to date stellar clusters, shifts only by about $0.1 \mathrm{mag}$. This implies that the WD age estimate is significantly less affected by the uncertainty on the distance modulus, which represents the main source of error in dating GCs with the method based on the turn off luminosity. Let us briefly recall the main WD evolutionary phases in order to explain the principal features in the WD isochrones and LFs. The evolution of WDs is initially characterized by a rapid drop of the luminosity and of the central temperature. The main energy loss characterizing this initial phase is the emission of neutrinos. The corresponding zone of the isochrone closely follows the track of a WD of fixed mass. Then, as a WD cools, the Coulomb interaction between ions becomes progressively greater and leads to the crystallization of carbon and eventually oxygen (Abrikosov 1960; Kirzhnits 1960; Salpeter 1961). Due to the latent heat released by the liquid-solid transition, the cooling rate slows temporarily (D'Antona \& Mazzitelli 1990; Fontaine et al. 2001). At the same time, the external convective zone penetrates the region where thermal conduction by degenerate electrons is very efficient. Such an occurrence (known as convective coupling), initially produces a further slowing down of the cooling timescale (see e.g. Chabrier et al. 2000; Fontaine et al. 2001). The steep increase in the WD luminosity function is the effect of such a decrease in the cooling rate. Finally, the WD enters the Debye regime, where the heat capacity decreases as $T^{3}$, thus depleting the WD of its main energy reservoir. As a consequence its luminosity quickly drops.

The morphology of the luminosity function reflects the variations of the cooling timescale: the LF is generally sparse at the brightest magnitudes and presents a steep rise, followed by a sharp cutoff, in the faintest part (see e.g. Fontaine et al. 2001).

The best way to date stellar systems through WDs is the analysis of their LF.

\section{The model}

The present WD theoretical tracks have been computed with the FRANEC (Chieffi \& Straniero 1989), a full evolutionary code that adopts the Henyey method and that is able to model the whole evolution of stars from the hydrostatic pre-main sequence phase up to the WD stage.

\subsection{Progenitors and starting model}

To provide the starting model for the WD evolution, we started from the ZAHB (Zero Age Horizontal Branch) model. The first model consists of a helium core (99\% of the total mass) and a

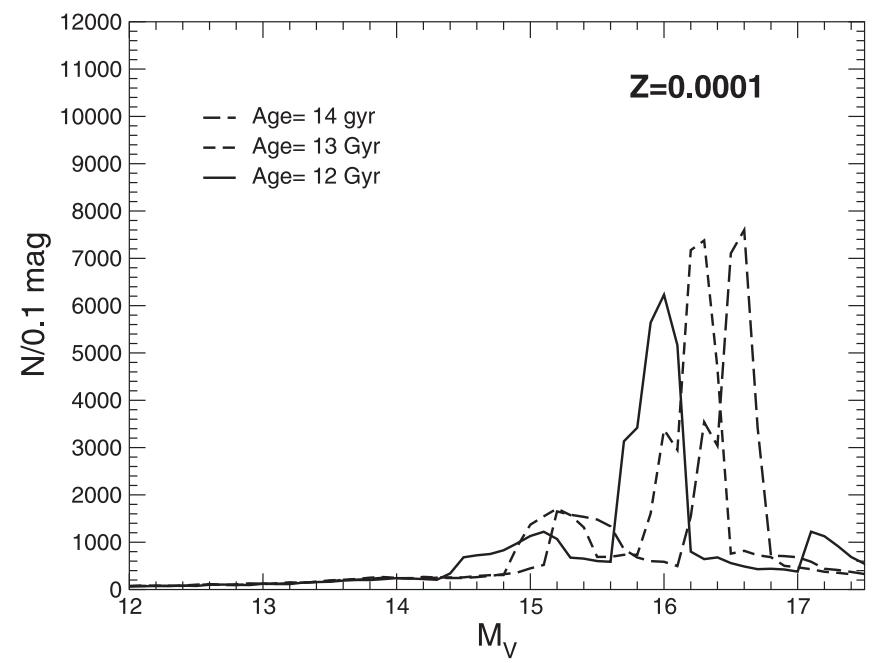

Fig. 2. Theoretical LFs for 12 (solid line), 13 (dashed line) and $14 \mathrm{Gyr}$ (long-dashed line). Each magnitude bin is $\Delta M_{V}=0.1 \mathrm{mag}$. The total number of WDs is 10000 .

thin hydrogen rich envelope $\left(Y=0.24 Z=10^{-4}\right)$. Then, we followed the evolution at constant mass, through the core Heburning. Due to the reduced envelope, at the central helium exhaustion the model rapidly moves towards the blue region of the HR diagram. During this transition the $\mathrm{He}$ - and the H-burning shells are eventually active. As discussed in Paper I, the model settles on the cooling sequence when the masses of both the Herich and the H-rich layers are reduced to certain critical values and the thermonuclear burning dies down.

We are aware that in the real world a DA C-O WD of a given mass can be formed through several different evolutionary paths, depending on the mass loss rate and on the initial chemical composition. This was one of the main points discussed in Paper I, where we studied three extreme cases, and we showed that the internal structure of the resulting WD is poorly affected by the different evolutionary histories (see e.g. Figs. 2 and 3 of Paper I). Moreover, the computation of the evolution through the thermally pulsing asymptotic giant branch (TP-AGB), although more realistic, is very time consuming. Thus, the method we have adopted represents a useful way to provide realistic WD starting models consistent with the evolution of the progenitors. In particular the chemical abundance profiles of carbon and oxygen in the core is largely independent of the evolutionary history. Obviously, the computation through the TP-AGB evolution should be preferred if the initial, hot phase of WD evolution is analyzed. In such an early phase of the cooling the outer layers still bear the record of the complex chemical abundance profile left by TP-AGB evolution, which is subsequently modified by diffusion. The metallicity of the progenitors determines the maximum value of the envelope mass, but this quantity does not significantly affect the cooling timescale (see Paper I). The only important quantity is the relation between the progenitor mass and the WD mass (initial-to-final mass relation) which is needed to correctly evaluate the time spent before the cooling sequence and, in turn, correctly locate a WD with a certain mass on the HR diagram (see Sect. 2.3). We stop the progenitor evolution when the newly formed WD reaches $\log L / L_{\odot} \sim 0$. At this point, the helium and hydrogen in the envelope are fully sorted, in order to reproduce the observed chemical stratification of the atmospheres of DA WDs. Several studies have indeed shown that in such a compact stars, where the surface gravity $g$ is of the order of $\sim 10^{8}-10^{9} \mathrm{~cm} \mathrm{~s}^{-2}$, the gravitational settling is efficient 


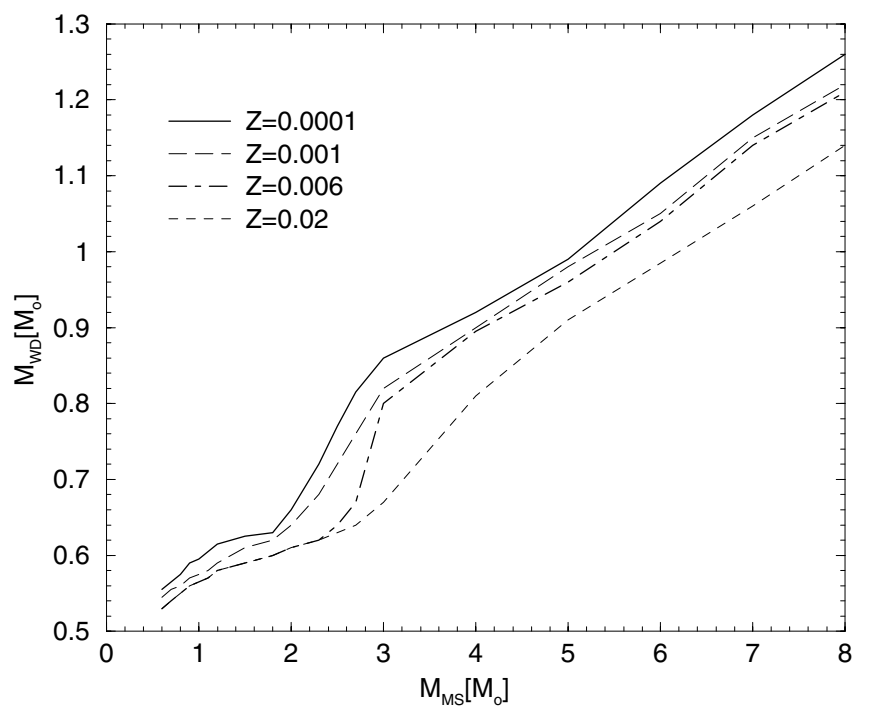

Fig. 3. Initial-to-final mass relationship for the four labeled metallicities from our stellar models. See text for details.

enough to account for the monoelemental feature of WD spectra (Fontaine \& Michaud 1979; Muchmore 1984; Paquette et al. 1986). The model obtained with this method is our starting point for the computation of the cooling sequence.

\subsection{Cooling tracks}

Let us recall the major features of the present models; for a detailed description see Paper I. We put considerable effort into updating the main input physics, namely the equation of state (EOS) for a high-density plasma, the radiative and conductive opacity and the model atmospheres. We updated and extended the EOS for a fully ionized plasma originally described by Straniero (1988). In particular, we have adopted the most recent results for the electrostatic corrections both in the liquid and in the solid phase (see Paper I for references). Concerning the Rosseland radiative opacity, we adopted the OPAL (Iglesias \& Rogers 1996) for high temperature $(\log T[\mathrm{~K}]>4.0)$ and the Alexander \& Ferguson (1994) molecular opacities for the low temperatures $(\log T[\mathrm{~K}]<4.0)$.

As we have shown in Paper I, for the cooling evolution of old WDs, the treatment of electron conduction in the partially degenerate regimes typical of the outer layers is complex. We have computed sets of theoretical cooling sequences and the related isochrones adopting the conductive opacity by Hubbard \& Lampe (1969, hereafter HL69), by Itoh and coworkers (Itoh et al. 1983; Mitake et al. 1984; and Itoh et al. 1993, hereafter I93), Potekhin (1999, hereafter P99; see also Potekhin et al. 1999), and combinations. The description of the different assumptions and the related effects on the cooling sequences of WDs is given in Sect. 5.

The computations of reliable cooling models rely on the choice of appropriate boundary conditions, namely the pressure at the base of the photosphere. As shown by Fontaine et al. (1974) and then confirmed by successive studies (see e.g. Tassoul et al. 1990; Hansen 1999) it is important to use a detailed model atmosphere to fix the surface boundary conditions. It is well known that one of the most important events in the evolution of old WDs, the coupling between the outer convective envelope and the isothermal degenerate core (see e.g.
Chabrier et al. 2000; Fontaine et al. 2001) is quite sensitive to the details of atmosphere stratification, in particular a gray approximation overestimate the inward penetration of the convection. The quoted coupling initially produces a sizeable slow down in the cooling evolution followed by a rapid drop in the luminosity. Since the occurrence of the convective coupling, the core, which is essentially the energy reservoir of the star, and the atmosphere, where the energy is radiated in space, are directly coupled. The pressure at the outer boundary, namely the layer where the optical depth $\tau=50$, has been obtained by interpolating on a grid of model atmospheres (Bergeron 2001) which cover a range of surface gravities between $10^{7.5}-10^{9} \mathrm{~cm} / \mathrm{s}^{2}$ and effective temperatures between $1500-100000 \mathrm{~K}$. A detailed treatment of the atmospheric structure is fundamental also for the prediction of the observable properties, the emergent spectrum and the photometric colors. As shown by Bergeron et al. (1995), when a WD with a pure hydrogen atmosphere cools down to $T_{\mathrm{e}} \sim 5000 \mathrm{~K}$ and the molecular recombination begins, the collisionally induced absorption (CIA) of $\mathrm{H}_{2}-\mathrm{H}_{2}$ progressively becomes the most important opacity source for the out-flowing infrared photons, so that the emergent spectrum departs from the blackbody appearance and the colors are blue shifted (see e.g. also Hansen 1999).

The present models take into account the liquid-solid phase transition and the related energy release (see e.g. Paper I) but neglect the effect of the phase separation during the crystallization of the core, i.e. the chemical redistribution of carbon and oxygen due to the shape of the phase diagram for this binary mixture (Stevenson 1980). We are interested in an evaluation of the relative effects on WD evolution of different physical aspects rather than to provide a reference standard model. Since the effect on WD evolution of chemical fragmentation has been already discussed several times in literature (see e.g. Segretain et al. 1994; Salaris et al. 1997; Montgomery et al. 1999; Isern et al. 1997, 2000) we do not further investigate this issue.

\subsection{Initial-to-final mass relation and initial mass function}

In order to compute isochrones, the age of the progenitor at the entrance of the cooling sequence must be added to the cooling time. This quantity depends on the mass and the chemical composition of the progenitor. The relationship between the main sequence mass and the final WD mass is still uncertain; it is sensitive to many poorly known phenomena, like the extention of the H-burning convective core, which affects the stellar lifetime, and the efficiency of the mass loss mechanism, particularly during the TP-AGB phase when the star loses the greatest fraction of its mass, which affects the final mass.

Semiempirical initial-to-final mass relations are only available for the chemical composition of the solar neighborhood and for the Magellanic Clouds (see e.g. Weidemann \& Koester 1983; Weidemann 1987, 2000; Herwig 1997). For the typical composition of the halo, the long distance and the large age limits a direct determination of the initial-to-final mass relation. Thus, in spite of the great uncertainty, we are forced to use theoretical relations. Figure 3 shows the initial-to-final mass relationship from the homogeneous set of stellar models of low and intermediate mass stars computed by Straniero et al. (1997) and Dominguez et al. (1999), with the same stellar evolutionary code used to provide the present set of WD models. The mass loss during the AGB phase has been obtained by best fitting the period-mass loss diagrams obtained by Whitelock et al. (2003). In stars with initial mass lower than $3 M_{\odot}$, an additional pre-AGB mass loss has been considered based on the Reimers formula with the parameter $\eta=0.4$. Table 1 reports the initial-to-final mass relations 
Table 1. Initial-to-final mass relationship for the four labeled metallicities from our stellar models. See text for details. Masses are in $M_{\odot}$.

\begin{tabular}{|c|c|c|c|c|c|c|c|c|c|c|c|c|c|c|c|c|c|}
\hline$\overline{\overline{M_{\mathrm{MS}}}}$ & 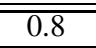 & $\overline{0.9}$ & $\overline{1.0}$ & 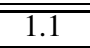 & 1.2 & 1.5 & 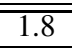 & 2.0 & 2.3 & 2.5 & 2.7 & 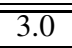 & 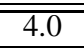 & 5.0 & 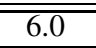 & $\overline{77.0}$ & 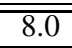 \\
\hline$M_{\mathrm{WD}}$ & 0.55 & 0.56 & 0.565 & 0.57 & 0.58 & 0.59 & 0.60 & $\begin{array}{c}Z=0.02 \\
0.61\end{array}$ & 0.62 & 0.63 & 0.64 & 0.67 & 0.81 & 0.91 & 0.985 & 1.06 & 1.14 \\
\hline & & & & & & & & $=0.006$ & & & & & & & & & \\
\hline$M_{\mathrm{WD}}$ & 0.55 & 0.56 & 0.565 & 0.57 & 0.58 & 0.59 & 0.60 & 0.61 & 0.62 & 0.64 & 0.67 & 0.80 & 0.895 & 0.96 & 1.04 & 1.14 & 1.21 \\
\hline & & & & & & & & $=0.001$ & & & & & & & & & \\
\hline$M_{\mathrm{WD}}$ & 0.56 & 0.57 & 0.575 & 0.58 & 0.59 & 0.61 & 0.62 & 0.64 & 0.68 & 0.72 & 0.76 & 0.82 & 0.90 & 0.98 & 1.05 & 1.15 & 1.22 \\
\hline & & & & & & & & $=0.0001$ & & & & & & & & & \\
\hline$M_{\mathrm{WD}}$ & 0.575 & 0.59 & 0.595 & 0.605 & 0.615 & 0.625 & 0.63 & 0.66 & 0.72 & 0.77 & 0.815 & 0.86 & 0.92 & 0.99 & 1.09 & 1.18 & 1.26 \\
\hline
\end{tabular}

shown in Fig. 3. Three distinct regions with different slopes may be distinguished. Concerning the solar metallicity, these three regions are: $M_{i}<3 M_{\odot}, 3 M_{\odot}<M_{i}<4.5 M_{\odot}$ and $M_{i}>4.5 M_{\odot}$, respectively. At lower metallicity the limit in mass of these three regions decreases, but the general features are conserved. For all the three regions the adopted mass loss prescription during the TP-AGB plays a crucial role in the determination of the final WD mass. Stars belonging to the first interval have a radiative core during the main sequence, or develop a small central convective zone $\left(\ll 0.5 M_{\odot}\right)$. Thus the mass of the hydrogen exhausted core $\left(M_{\mathrm{H}}\right)$ at the first thermal pulse depends on the efficiency of the H-burning shell operating during the RGB phase and the following He-burning phase, and on the corresponding evolutionary time scales. These parameters are very similar for all these low mass stars so that their $M_{\mathrm{H}}$ at the first thermal pulse is practically the same $\left(\sim 0.55 M_{\odot}\right.$, see e.g. Fig. 15 of Dominguez et al. 1999). Then, the most important phenomenon that influences the final WD mass of these stars is the mass loss, taking place during the RGB and AGB phases, which erodes the envelope and limits the further growth of $M_{\mathrm{H}}$ during the thermally pulsing AGB. At variance, stars belonging to the second zone develop a sizeable convective core when they burn the central $\mathrm{H}$. Thus, $M_{\mathrm{H}}$ at the first thermal pulse is mainly determined by the maximum extension attained by the convective core during the main sequence. In this case, the pre-AGB mass loss have a negligible effect on the final mass, while that in the TP-AGB phase is, once again, crucial. Similar considerations are valid for the stars belonging to the third region, but, in this case, the growth of the core is also limited by the occurrence of the II dredge-up, during the earlyAGB phase. During the TP-AGB phase the recursive occurrence of a deep III dredge-up brings inward fresh hydrogen and puts backward the outer edge of the hydrogen exhausted core, thus preventing a substantial increase of $M_{\mathrm{H}}$. For this reason, the final mass is only slightly larger than $M_{\mathrm{H}}$ at the beginning of the TP-AGB phase. As shown in Fig. 20 of Dominguez et al. (1999) there is a general agreement among the available predictions for $M_{\mathrm{H}}$ at the first thermal pulse as a function of the initial mass. The most important consequence of the assumption of a sizeable convective core overshoot is the decrease of the maximum mass able to produce a C-O WD ( $M_{\text {up }}$, Bertelli et al. 1985). In order to compute WD LFs and synthetic color-magnitude diagrams (CMDs) we also need the initial mass function (IMF) for the progenitor stars. In the following, if not explicitely stated, we have adopted a single power law IMF $\left(\mathrm{d} N / \mathrm{d} M_{i} \propto M_{i}^{-\alpha}\right)$, with a Salpeter exponent $(\alpha=2.3)$. The effect of varying both the slope

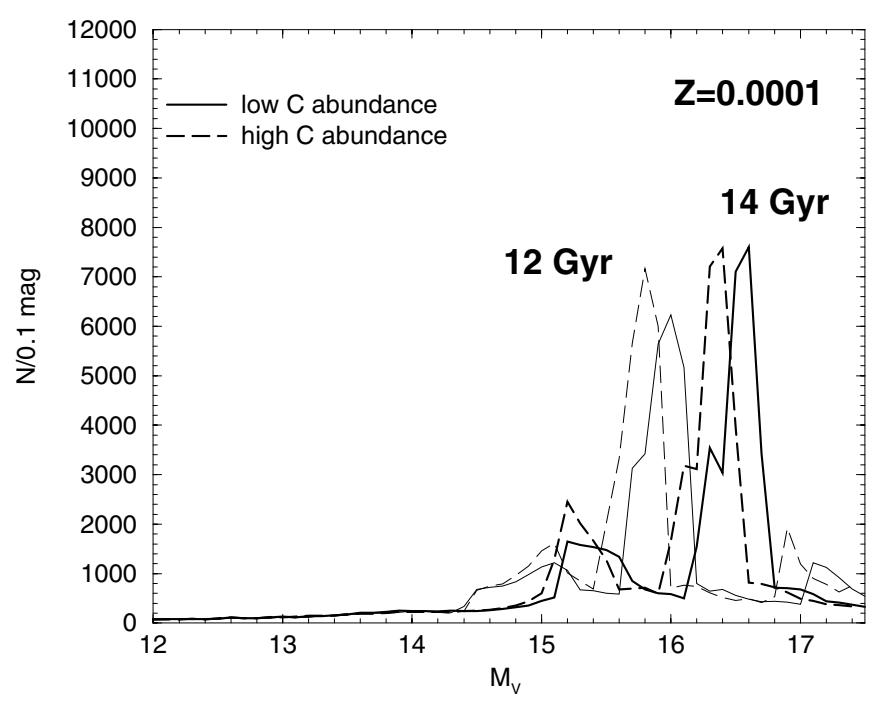

Fig. 4. Theoretical LFs for 12 (thin lines) and 14 Gyr (thick lines) for WDs with low (solid lines) and high (dashed lines) C abundance.

of the IMF and the initial-to-final mass relationship will be discussed in Sect. 7.

\section{Carbon and oxygen abundances in the core}

The thermal energy of a cool WD is almost entirely stored in the carbon and oxygen ions of the core. The cooling rate depends sensitively on the relative amount of carbon and oxygen. Carbon has an higher specific heat than oxygen, simply because the number of ions in a gram of $\mathrm{C}$ is larger. Thus, the cooling rate depends sensitively on the $\mathrm{C} / \mathrm{O}$ ratio: the higher the carbon abundance in the core, the slower the cooling of the WD (Salaris et al. 1997; Chabrier et al. 2000; Paper I). In addition, since carbon crystallizes later than oxygen, the release of the latent heat and the onset of convective coupling are delayed when carbon is overabundant.

The chemical abundance profiles in the core of a $\mathrm{C}-\mathrm{O}$ WD are the result of the helium burning that occurred in the core and in the shell of the progenitor star. As is well known, initially the $3 \alpha$ reaction produces carbon and afterwards the ${ }^{12} \mathrm{C}(\alpha, \gamma){ }^{16} \mathrm{O}$ synthesizes oxygen. The final chemical stratification in the core of a WD depends on the relative efficiency of these two reactions. In particular, the reaction rate of the ${ }^{12} \mathrm{C}(\alpha, \gamma){ }^{16} \mathrm{O}$ at an 


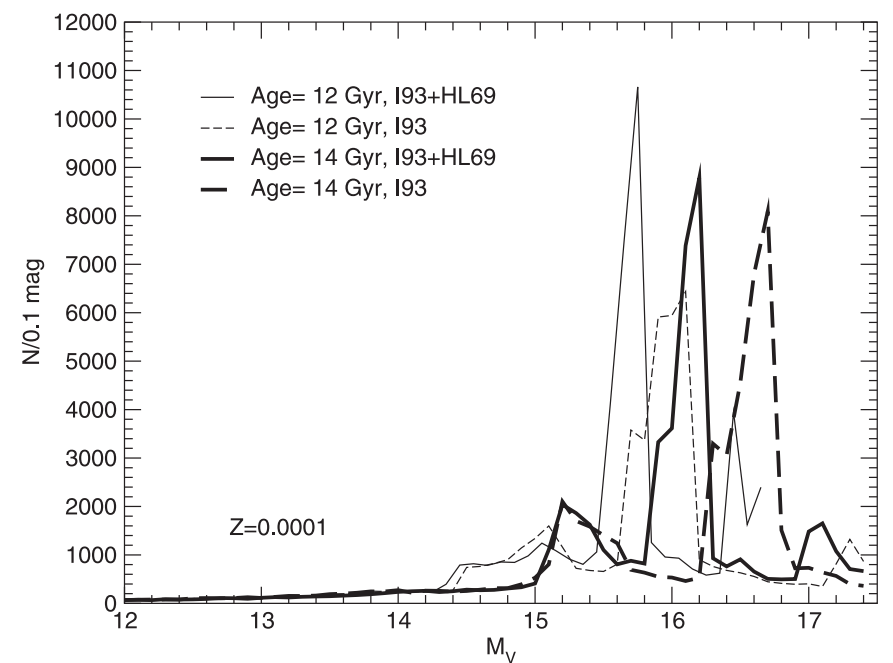

Fig. 5. Theoretical LFs for 12 (thin lines) and 14 Gyr (thick lines) for WD models computed adopting the conductive opacity of Itoh and coworkers (I93) in the fully degenerate regime $\left(\theta=T_{\mathrm{F}} / T<0.1\right)$ and Hubbard \& Lampe (HL69) in the partially degenerate one $(\theta>0.1)$ (I93+HL69, solid lines) and I93 in the whole structure (I93, dashed lines).

energy relevant for the stellar He-burning (about $300 \mathrm{KeV}$ ) is only known within a factor of 2 (see e.g. Buchman 1996; Kunz et al. 2002). The treatment of the convective mixing during the core He-burning phase also affects the predicted $\mathrm{C} / \mathrm{O}$ ratio (see Imbriani et al. 2001; Straniero et al. 2003).

In Paper I we analyzed the changes of the cooling timescale of a $0.6 M_{\odot}$ CO WD caused by the variation of the ${ }^{12} \mathrm{C}(\alpha, \gamma){ }^{16} \mathrm{O}$ reaction rate, within the present error bars, and by different prescriptions for the core convection.

In this section we extend that analysis to masses from 0.5 to $0.9 M_{\odot}$ and calculate the corresponding isochrones and LFs. Then, two sets of WD cooling tracks were obtained for two different $\mathrm{C}-\mathrm{O}$ chemical stratifications in the core, the low carbon abundance model with a central carbon mass fraction of about 0.2 and the high ones with 0.50 , a range that roughly represents the present uncertainty in the theoretical prediction. The former value appears in better agreement with the abundance derived from the power spectra of variable WDs (Metcalfe et al. 2001), although the feasibility of this kind of measurement has been questioned (Fontaine \& Brassard 2002).

Figure 4 shows the 12 (thin lines) and 14 Gyr (thick lines) LFs for WDs with low (solid lines) and high (dashed lines) C abundance. As expected, due to the lower heat capacity of the carbon-poor material, the cooling time is shorter in these models than the cooling time of the carbon-rich models. As a consequence, the WD LF peak occurs at lower luminosity. The effect of the present uncertainty on the chemical profile in the core is sizeable.

Taking into account that the position of the WD LF peak is a very sensitive function of the age $\left(\Delta M_{V} / \Delta\right.$ Age $\sim 0.3 \mathrm{mag} / \mathrm{Gyr}$ for GCs ages), the poor determination of the $\mathrm{C}$ abundance in the core translates directly into an uncertainty of about $0.6 \mathrm{Gyr}$ in the inferred GC age.

\section{Conductive opacity}

The electrons in the core of a WD are fully degenerate. In these conditions the energy transfer is largely dominated by electron conduction whose efficiency is high enough to keep the core

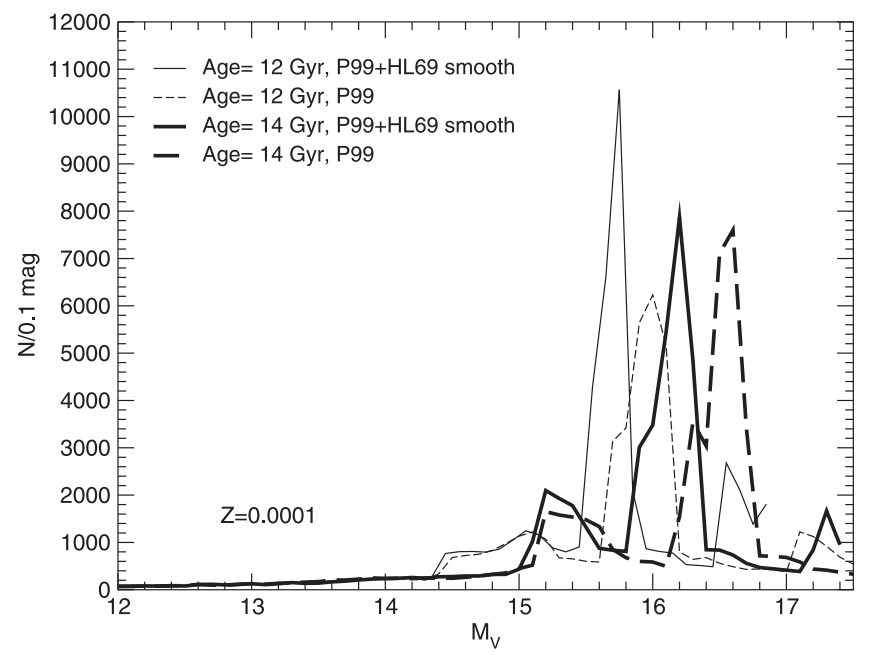

Fig. 6. Theoretical LFs for 12 (thin lines) and 14 Gyr (thick lines) for WD models computed adopting the conductive opacity of Potekhin (P99) and HL69 in the regions where, respectively, $\theta<0.1$ and $\theta>1$, and a linear interpolation in the transition zone (P99+HL69 smooth, solid lines) and the ones computed adopting the P99 conductive opacity in the whole structure (dashed lines).

almost isothermal. On the contrary, in the mantel (He-rich) and in the envelope (H-rich) the electrons are only partially or not degenerate, so that thermal conduction is less efficient. In bright WDs, the thin envelope is the most opaque region of the WD, a kind of insulating layer which regulates the temperature decrease of the core. The progressive development of the external convection penetrating the partially degenerate region in cool WDs causes the already mentioned convective coupling. Therefore, it is of primary importance for the description of the cooling process to understand the detailed treatment of the energy transport through the external layers (see e.g. D'Antona \& Mazzitelli 1990; Fontaine et al. 2001).

In our knowledge, there are only two published conductive opacities suitable for partially degenerate regimes, the pioneering study by Hubbard \& Lampe (1969, hereafter HL69) and Potekhin (1999, hereafter P99; see also Potekhin et al. 1999) ${ }^{1}$. Regarding the fully degenerate regime, in addition to the just mentioned works, there are also the fundamental contributions by Itoh and coworkers (Itoh et al. 1983; Mitake et al. 1984; and Itoh et al. 1993, hereafter I93).

As discussed in detail in Paper I, the HL69, although nominally valid, should not be adopted in regions with a high Coulomb coupling parameter $\left(\Gamma>10\right.$, where $\Gamma=(Z e)^{2} / k_{B} T a$ and $a$ is the interionic distance) due to an outdated treatment of the liquid-solid transition. On the other hand, the I93 computations are strictly valid only in the completely degenerate regimes $\left(\theta=T_{\mathrm{F}} / T<0.1\right.$, where $T_{\mathrm{F}}$ is the electronic Fermi temperature), in fact they underestimate the contribution of the electron-electron interactions which is not negligible in the partially degenerate regimes. The only conductive opacity computations suitable for the whole WD structure and evolution are those by P99.

In Paper I we showed the dramatic effect on the evolution of a $0.6 M_{\odot}$ WD of different assumptions for the conductive opacity in the thin envelope. At $\log L / L_{\odot}=-5.5$ the adoption of the I93 in the whole WD structure leads to an age $17 \%$ younger than

1 We have derived conductive opacity tables by means of the Fortran codes provided by Potekhin and available at the web site (http://www.ioffe.rssi.ru/astro/conduct/conduct.html). 
that obtained by using HL69 in the partially degenerate regime. In this section we extended that analysis to evaluate the effect on the WD isochrones and LFs of different assumptions on the conductive opacity.

We have computed five sets of cooling tracks with masses in the range $0.5-0.9 M_{\odot}$ and the related set of isochrones and LFs. Figure 5 shows the theoretical LFs for 12 (thin lines) and 14 Gyr (thick lines) for WD models computed adopting the conductive opacity of Itoh and coworkers (I93) in the fully degenerate regime $(\theta<0.1)$ and Hubbard \& Lampe (HL69) in the partially degenerate one $(\theta>0.1)$ (I93+HL69, solid lines) and I93 in the whole structure (I93, dashed lines). Notwithstanding that the two pairs of sets differ only in the conductive efficiency adopted in the partially degenerate regime, roughly corresponding to the thin external layer, whose mass is less than $1 \%$ of the WD mass, the effect is very large causing a shift in the position of the LF peak of about $0.3 \mathrm{mag}$ for $12 \mathrm{Gyr}$ and $0.5 \mathrm{mag}$ for 14 Gyr, which means roughly a difference in age of 1 Gyr and $1.6 \mathrm{Gyr}$, respectively.

Itoh suggested to use a different match between the I93 and the HL69 conductive opacity (private communication). Thus, we have calculated an additional set of cooling sequences, here referred to as I93+HL69 smooth, where I93 is used for $\theta<0.1$, HL69 for $\theta>1$ and a linear interpolation is used between the two for $0.1<\theta<1$. The variation of the WD LF peak is less than 0.1 mag. In addition, we have computed cooling models adopting the conductive opacity of Potekhin (P99) and HL69 in the regions where, respectively, $\theta<0.1$ and $\theta>1$, and a linear interpolation in the transition zone (here referred as P99+HL69 smooth). To compute the conductive opacity of Potekhin we use Fortran codes provided by Potekhin. The differences are small, as expected from a previous analysis presented in Paper I, because the P99 predictions are in good agreement with the I93 ones where this latter is nominally valid.

Figure 6 shows the LFs for 12 (thin lines) and 14 Gyr (thick lines) for the just presented "P99+HL69 smooth" WD models (solid lines) and the ones computed adopting the P99 conductive opacity in the whole structure (dashed lines). The discrepancy between the two sets of models is quite large, for the presented ages the shift of the WD LF peak is about 0.4 mag, roughly corresponding to $1.3 \mathrm{Gyr}$. As in the case shown in Fig. 5, this result proves again the extreme sensitivity of the computed cooling times and thus of the theoretical WD LFs to the treatment of conductive opacity in the very thin $\left(M_{\mathrm{env}} / M_{\mathrm{WD}}<0.01\right)$ envelope, where electrons are only partially degenerate. To our knowledge, Potekhin provides the only homogeneous conductive opacity computations suitable for the description of the whole WD structure available in the literature. On the other hand, these results for nondegenerate plasmas, as stated by Potekhin himself, "are based on a continuation from the degenerate domain (using Fermi-Dirac averaging) and can be considered as order-of-magnitude estimates." (see e.g. description at http://www . ioffe.rssi.ru/astro/conduct/conduct. html). To provide firmer predictions of WD cooling times we need more precise treatments of electron conduction for partially degenerate regimes.

At present the choice of the prescription adopted for the conductive opacity affects the age estimate of GCs at the level of $10 \%$.

\section{Metal abundance}

In Paper I we computed the evolution of two $0.6 M_{\odot}$ WD models produced by progenitor stars with very different metallicity,

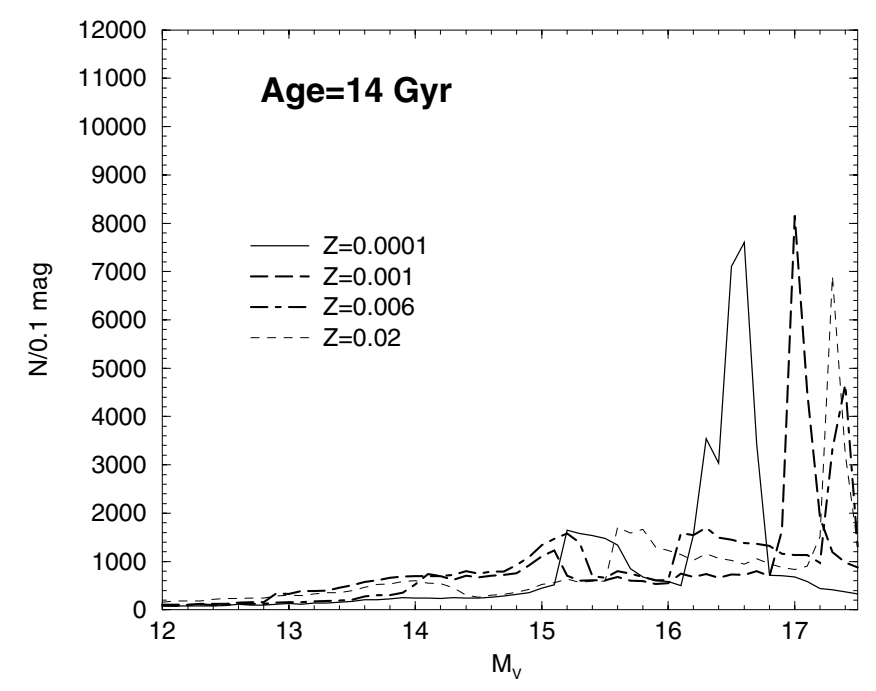

Fig. 7. Theoretical LFs of 14 Gyr for the four labeled metallicities.

respectively $Z=0.0001$ and 0.02 . The difference between the two cooling times are almost negligible (see e.g. Fig. 5, in Paper I), about $2 \%$ at the faint end of our computations $\left(\log L / L_{\odot}=-5.5\right)$.

The larger the metallicity, the thinner the external H-layer, because the $\mathrm{H}$ burning efficiency increases with a larger amount of CNO. Due to the compact nature of these stellar remnants the gravitational settling is so efficient as to produce an envelope constituted by two layers of almost pure elements, i.e. a buffer of He surrounded by a very thin $\mathrm{H}$ surface envelope.

On the contrary, the WD isochrones and LFs are considerably affected by metallicity variations because, as is well known, the pre-WD evolutionary time scale is a sensitive function of the original chemical abundance. In addition, the initial-to-final mass relation changes with metallicity.

In order to estimate the effect of the assumed chemical abundance, we computed four sets of isochrones and the related LFs for metallicities covering a range suitable for population I and II stars, i.e. $Z=0.0001,0.001,0.006$ and 0.02 . As already mentioned, we adopted the pre-WD evolutionary times and the initial-to-final mass relationship (see e.g. Fig. 3) from the homogeneous set of stellar models of low and intermediate mass stars by Dominguez et al. (1999) and Straniero et al. (1997).

Figure 7 shows the theoretical WD LFs for an age of $14 \mathrm{Gyr}$ and the four labeled metallicities. The position of the peak of the WD LF is a sensitive function of the metal content of the progenitor stars. Between $Z=0.0001$ and $Z=0.001$, there is a difference in the visual magnitude of the peak of about 0.4 mag, the same between $Z=0.001$ and $Z=0.006$. Thus, in order to use WDs to date GCs, one should compute theoretical isochrones and LFs with the cluster metallicity. For galactic GCs the typical error on $[\mathrm{Fe} / \mathrm{H}]$ is of the order of $0.1 \mathrm{dex}$. This means that the typical uncertainty on the metallicity measurements only slightly affects the estimates of the GC ages based on the WD cooling sequences, provided that WD isochrones and LFs have been computed with the nominal value of the cluster metal abundance.

\section{Initial-to-final mass relation and IMF}

Figure 8 shows some of the most recent initial-to-final mass relations for the chemical composition typical of the solar neighborhood. Weidemann (2000, filled circles) derived the WD mass by comparing theoretical WD evolutionary models with 


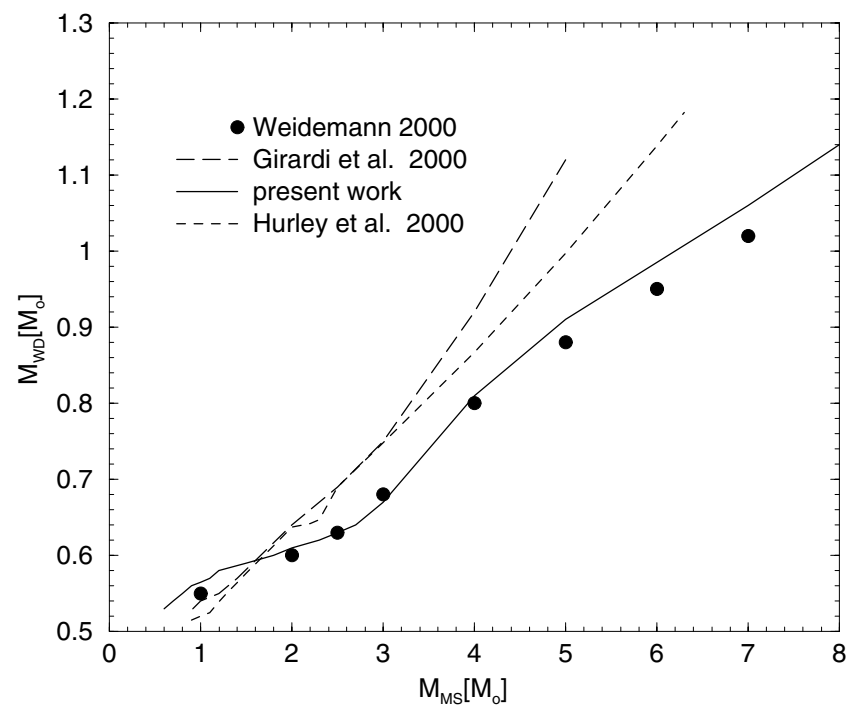

Fig. 8. Initial-to-final mass relations for the solar chemical composition from: Weidemann (2000, filled circles); Girardi et al. (2000, dashed line); our relation (solid line); Hurley et al. (2000, short dashed line).

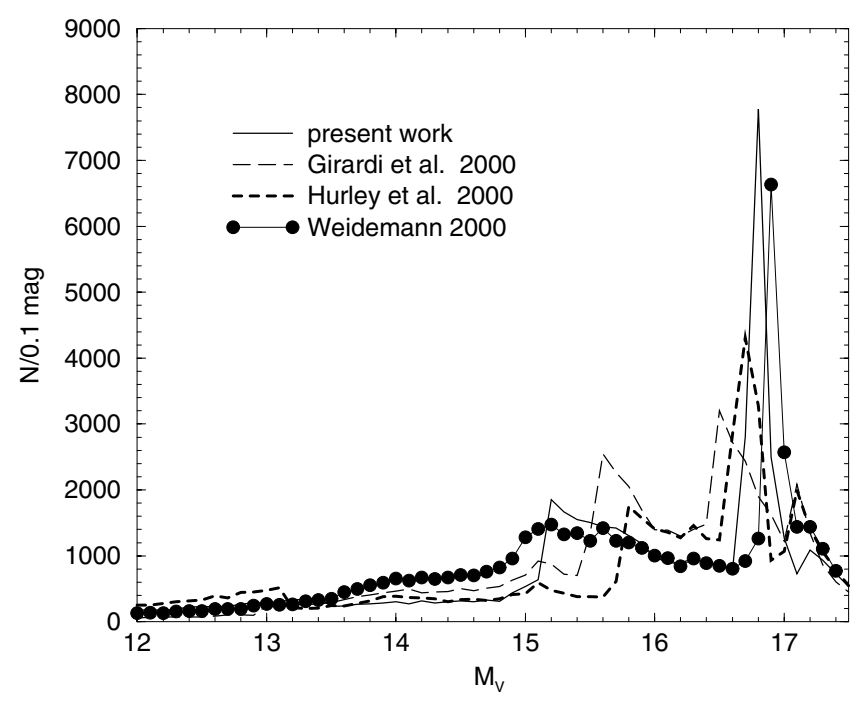

Fig. 9. Theoretical WD LFs for $12 \mathrm{Gyr}$ and different initial-to-final mass relations for the solar chemical composition from: Weidemann (2000, filled circles); Girardi et al. (2000, dashed line); our relation (solid line); Hurley et al. (2000, short dashed line).

the surface gravities and effective temperatures inferred from spectroscopy. Then the initial mass is obtained by comparing the cluster turn off luminosity with stellar models of different masses (see e.g. Weidemann \& Koester 1983); thus also the semiempirical method depends on some theoretical assumptions, like the convective core overshoot. As mentioned above and as can be seen in Fig. 8, the initial-to-final mass relation is still quite uncertain, even in the case of typical solar chemical composition.

Girardi et al. (2000) rely on synthetic AGB models. This means that the evolution of the AGB stellar properties (the core mass, the total mass, the luminosity or the effective temperature) is obtained by semiempirical prescriptions, instead of solving the stellar structure equations (see e.g. Groenewegen \& de Jong 1993; Bertelli et al. 1994; Marigo et al. 1996, 1998; Girardi \& Bertelli 1998). The Hurley et al. (2000) relation has been computed with the SSE package, kindly provided by Hurley, which uses analytic formulae derived by fitting the stellar models

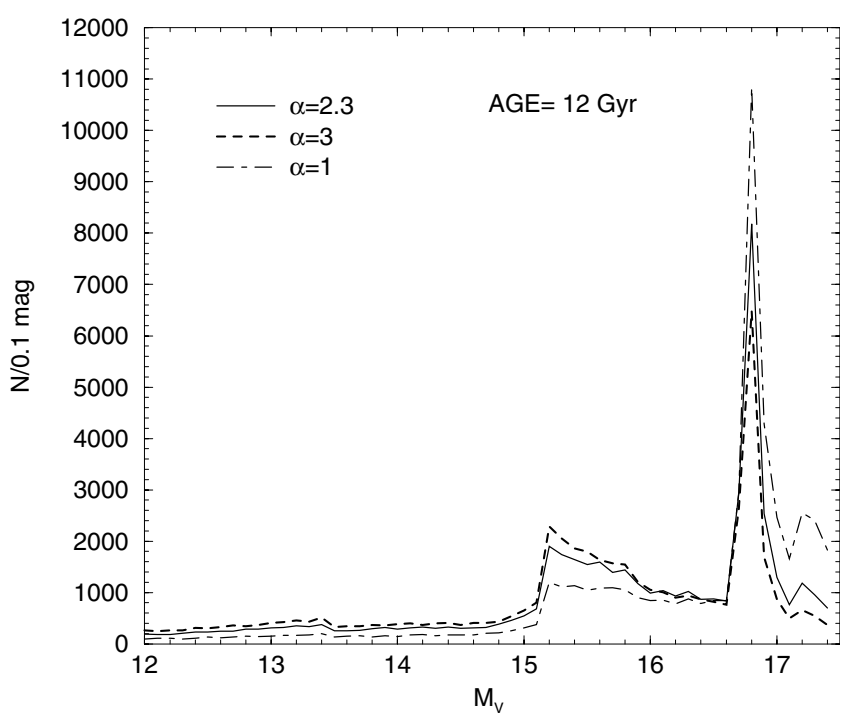

Fig. 10. Theoretical WD LFs for $12 \mathrm{Gyr}$ and solar metallicity. Three different slopes of the IMF $\left(\propto M^{-\alpha}\right)$ have been adopted: $\alpha=2.3$ (solid line); $\alpha=3$ (dashed line); $\alpha=1$ (dot-dashed line).

computed by Pols et al. (1995). Our prescription for the initialto-final mass relation is based on full stellar evolution computations, from the pre-MS up to the AGB tip (Straniero et al. 1997; Dominguez et al. 1999; Straniero et al. 2005). The pre-AGB mass loss is based on the Reimers' formula $(\eta=0.4)$, and an empirical calibration of the mass loss-period relation is used for the AGB evolution (see Straniero et al. 2005, for details). Thus, the three curves reported in Fig. 8 are representative of the different methods commonly used to derive the initial-to-final mass relation.

Figure 9 shows the WD LFs related to the initial-to-final mass relations described in Fig. 8. In all cases we have adopted the same set of cooling evolutionary models and the same slope of the IMF. We derived the pre-WD ages from the homogeneous set of stellar models of low and intermediate mass stars by Straniero et al. (1997) and Dominguez et al. (1999), except for the Girardi et al. (2000) and the Hurley et al. (2000) relations, for which we used the life times provided by their own stellar models. The substitution of our relation by the Weidemann (2000) one produces a shift of the peak of about $0.1 \mathrm{mag}$, roughly corresponding to $0.3 \mathrm{Gyr}$. The same shift, but in the opposite direction, is produced by substituting our relation with the Hurley et al. (2000) one. A larger effect is determined by changing our relation with the Girardi et al. (2000) one; in this case the peak shifts by about $0.3 \mathrm{mag}$, which translates into an age error of 1 Gyr. To illustrate the effect of a different mass distribution of the progenitor stars, we have computed WD LFs adopting a single power law IMF $\left(\mathrm{d} N / \mathrm{d} M_{i} \propto M_{i}^{-\alpha}\right)$ varying the slope within a wide range, i.e. $\alpha=1,2.3$ and 3 . Figure 10 shows the results; as expected the variation of the slope of the IMF affects only the height of the peak, but leaves its position practically unchanged.

\section{Conclusions}

WD cosmochronology is becoming a feasible tool for dating stellar clusters. In this paper we have analyzed the accuracy of the available calibration of the WDs age, by computing various sets of WD isochrones and LFs under different prescriptions for the theoretical ingredients. We discussed the effect on the WD LFs, and thus on the globular cluster age derived with these 
compact objects, of the assumed conductive opacity, $\mathrm{C}-\mathrm{O}$ abundance in the core, and initial-to-final mass relation and IMF.

The main effect is caused by the adopted conductive opacity, in particular in the partially degenerate regime characteristic of the thin outer layer. For the range of ages typical of galactic GCs the conductive opacity affects the dating by the order of $10 \%$.

Concerning the $\mathrm{C}-\mathrm{O}$ amount in the core, the uncertainty in the age is less than 5\%. Moreover, this contribution will further decrease in the near future thanks to experiments to measure the ${ }^{12} \mathrm{C}(\alpha, \gamma){ }^{16} \mathrm{O}$ reaction rate at the energy of interest.

The IMF does not affect the position of the peak and consequently the estimated ages. At variance, the initial-to-final mass relation plays an important role in dating globular clusters because it significantly affects the shape of the WD LF, in particular the position of the peak. The uncertainty on the age can reach $8 \%$.

Finally, given the sensitive dependence of the evolution of the WD progenitors on the metal content, in order to use WDs to date GCs, one has to compute WD isochrones and LFs for the metallicity of the cluster. In fact, between $Z=0.0001$ and 0.001 and between $Z=0.001$ and 0.006 , the difference in the inferred age is, for the range of interest, of the order of $10 \%$.

Acknowledgements. We thank P. Bergeron for kindly providing us the model atmosphere and N. Itoh and S. Shore for the many helpful comments. We are grateful to J. R. Hurley for kindly providing us the SSE package.

\section{References}

Abrikosov, A. A. 1960, Zh. Eksp. Teor. Fiz., 39, 1798

Alexander, D. R., \& Ferguson, J. W. 1994, ApJ, 437, 879

Benvenuto, O. G., \& Althaus, L. G. 1999, MNRAS, 303, 30

Bergeron, P. 2001, private comunication

Bergeron, P., Saumon, D., \& Wesemael, F. 1995, ApJ, 443, 764

Bergeron, P., Leggett, S. K., \& Ruiz, M. T. 2001, ApJS, 133, 413

Bertelli, G., Bressan, A. G., \& Chiosi, C. 1985, A\&A, 150, 33

Bertelli, G., Bressan, A. G., Chiosi, C., Fagotto, F., \& Nasi, E. 1994, A\&AS, 106,275

Buchmann, L. 1996, ApJ, 468, 127

Chabrier, G., Brassard, P., Fontaine, G., \& Saumon, G. 2000, ApJ, 543, 216

Chieffi, A., \& Straniero, O. 1989, ApJS, 71, 47

Cool, A., Piotto, G., \& King, I. R. 1996, ApJ, 468, 655

D'Antona, F., \& Mazzitelli, I. 1990, ARA\&A, 28, 139

De Marchi, G., Paresce, F., Straniero, O., \& Prada Moroni, P. G. 2004, A\&A, 415,971

Dominguez, I., Chieffi, A., Limongi, M., \& Straniero, O. 1999, ApJ, 524, 226

Dominguez, I., Hoflich, P., \& Straniero, O. 2001, ApJ, 557, 279

Fontaine, G., \& Brassard, P. 2002, ApJ, 581, L33

Fontaine, G., \& Michaud, G. 1979, ApJ, 231, 826

Fontaine, G., Brassard, P., \& Bergeron, P. 2001, PASP, 113, 409

Fontaine, G., Van Horn, H. M., Bohm, K.-H., \& Grenfell. T. C. 1974, ApJ, 193, 205

Fusi Pecci, F., \& Renzini, A. 1979, in Astronomical Uses of the Space Telescope, ed. F. Macchetto, F. Pacini, \& M. Tarenghi, Geneva: ESO 181
Girardi, L., \& Bertelli, G. 1998, MNRAS, 300, 533

Girardi, L., Bressan, A., Bertelli, G., \& Chiosi, C. 2000, A\&ASS, 141, 371

Groenewegen, M. A. T., \& de Jong, T. 1993, A\&A, 267, 410

Hansen, B. M. S. 1999, ApJ, 520, 680

Hansen, B. M. S., Brewer, J., Fahlman, G. G., et al. 2002, ApJ, 574L, 155

Herwig, F. 1997, in Proc. 10th European Workshop on White Dwarfs, ed. J. Isern, M. Hernanz, \& E. Gracia-Berro (Dordrecht: Kluwer), 63

Herwig, F. 2005, ARA\&A, 43, 435

Hubbard, W. B., \& Lampe, M. 1969, ApJS, 18, 297

Hurley, J. R., Pols, O. R., \& Tout, C. A., MNRAS, 315, 543

Iben, I. Jr., Kaler, J. B., Truran, J. W., \& Renzini, A. 1983, ApJ, 264, 605

Iben, I. Jr., \& MacDonald, J. 1995, in White Dwarf, ed. D. Koester, \& K. Werner (Heidelber: Springer-Verlag) LNP 443, 48

Iglesias, A., \& Rogers, F. J. 1996, ApJ, 464, 943

Imbriani, G., Limongi, M., Gialanella, L., Straniero, O., \& Chieffi, A. 2001, ApJ, 558,903

Isern, J., Mochkovitch, R., Garcia-Berro, E., \& Hernanz, M. 1997, ApJ, 485, 308

Isern, J., Garcia-Berro, E., Hernanz, M., \& Chabrier, G. 2000, ApJ, 528, 397

Itoh, N., Mitake S., Iyetomi, H., \& Ichimaru, S. 1983, ApJ, 273, 774

Itoh, N., Hayashi, H., \& Kohyama, Y. 1993, ApJ, 418, 405

Kirzhnits, D. A. 1960, Soviet Phys.-JETP, 11, 365

Koester, D., \& Chanmugam, G. 1990, Rep. Prog. Phys., 53, 837

Kunz, R., Fey, M., Jaeger, M., et al. 2002, ApJ, 567, 643

Marigo, P., Girardi, L., \& Chiosi, C. 1996, A\&A, 316, L1

Marigo, P., Bressan, A., \& Chiosi, C. 1998, A\&A, 331, 564

Metcalfe, T. S., Winget, D. E., \& Charbonneau, P. 2001, ApJ, 557, 1021

Mitake S., Ichimaru, S., \& Itoh, N. 1984, ApJ, 277, 375

Monelli, M., Corsi, C. E., Castellani, V., et al. 2005, ApJ, 621, L117

Montgomery, M. H., Klumpe, E. W., Winget, D. E., \& Wood, M. A. 1999, ApJ, 525,482

Muchmore, D. 1984, ApJ, 278, 769

Paquette, C., Pelletier, C., Fontaine, G., \& Michaud, G. 1986, ApJS, 61, 197

Paresce, F., De Marchi, G., \& Romaniello, M. 1995, ApJ, 440, 216

Pols, O. R., Schroder, K. P., Hurley, J. R., Tout, C. A., \& Eggleton, P. P. 1998, MNRAS, 298, 525

Potekhin, A. Y. 1999, A\&A, 351, 787

Potekhin, A. Y., Baiko, D. A., Haensel, P., \& Yakovlev, D. G. 1999, A\&A, 346, 345

Prada Moroni, P. G., \& Straniero, O. 2002, ApJ, 581, 585

Renzini, A., Bragaglia, A. Ferraro, F. R., et al. 1996, ApJ, 465L, 23

Richer, H. B., Fahlman, G. G., Ibata, R. A., et al. 1997, ApJ, 484, 741

Salaris, M., Dominguez, I., Garcia-Berro, E., et al. 1997, ApJ, 486, 413

Salaris, M., Garcia-Berro, E., Hernanz, M., Iserm, J., \& Saumon, D. 2000, ApJ, 544,1036

Salpeter, E. E. 1961, ApJ, 134, 669

Schmidt, M. 1959, ApJ, 129, 243

Segretain, L., Chabrier, G., Hernanz, M., et al. 1994, ApJ, 434, 641

Stevenson, D. J. 1980, J. Phys. Suppl., 41, C2

Straniero, O. 1988, A\&AS, 76, 157

Straniero, O., Chieffi, A., \& Limongi, M. 1997, ApJ, 490, 425

Straniero, O., Dominguez, I., Imbriani, G., \& Piersanti, L. 2003, ApJ, 583, 878

Straniero, O., Gallino, R., \& Cristallo, S. 2005, Nucl. Phys. A, in press

Tassoul, M., Fontaine, G., \& Winget, D. E. 1990, ApJS, 72, 335

Von Hippel, T., \& Gilmore, G. 2000, AJ, 120, 1384

Weidemann, V. 1987, A\&A, 188, 74

Weidemann, V. 2000, A\&A, 363, 647

Weidemann, V., \& Koester, D. 1983, A\&A, 121, 77

Whitelock, P. A., Feast, M. W., van Loon, J. T., \& Zijlstra, A. A. 2003, MNRAS, 342, 86

Zoccali, M., Renzini, A., Ortolani, S., et al. 2001, ApJ, 553, 733 